Tesis. Año 14, 13(16), 2020, 127-154

\title{
La complejidad de la coginición humana: creer
}

\author{
Daniel Alejandro Castro Figueroa \\ dancasfig@yahoo.es
}

\section{Resumen}

El trabajo a continuación intenta ampliar la perspectiva sobre tipos de creencias en tanto que tradicionalmente solo se ha tomado en cuenta a las creencias del tipo consciente y lingüístico. En otras palabras, se ha asumido que la cognición humana solo crea creencias a partir de nuestra capacidad para producir lenguaje. Contrario a esto, el presente artículo sugiere que la cognición implica un espectro de creencias que también abarca a aquellas que no son lingüísticas ni conscientes, además de que las emociones son creencias en tanto programas proposicionales de supervivencia. Para lograr este objetivo, se utilizarán teorías de la filosofía de la mente, el psicoanálisis contemporáneo, la psicología budista y la neurociencia. A partir de todo lo anterior, la cantidad de creencias que poseemos es bastante más amplia que aquellas de las cuales somos conscientes lingüísticamente.

Palabras clave: creencia, cognición, filosofía de la mente, consciencia, lingüística.

\section{Abstract}

The following work attempts to broaden the perspective regarding beliefs considering that traditionally only beliefs of the conscious and linguistic type have been considered. In other words, it has been assumed that human cognition only creates beliefs from our ability to produce language. Contrary to this, the article suggests that cognition implies a spectrum of beliefs that also encompasses those that are neither linguistic nor conscious, and that emotions are beliefs as propositional survival programs. In order to accomplish this, theories of the philosophy of the mind, contemporary psychoanalysis, Buddhist psychology and neuroscience are treated. Taking all in consideration, we can assure that the amount of beliefs we have is much wider than those of which we are linguistically aware.

Key words: belief, cognition, philosophy of mind, consciousness, linguistics. 


\section{La complejidad de la coginición humana: creer}

\section{Introducción}

En Filosofía de la Mente, aún no se ha considerado cabalmente la amplitud del fenómeno mente-cerebro. Los debates sobre la mente giran en torno la consciencia lingüística únicamente. En particular, esto ha repercutido en el concepto de "creencia". Grosso modo, esta palabra se define como el hecho de que alguien considere que una proposición es verdad. En tal sentido, usualmente se antepone el hecho de que podemos enunciar las proposiciones, por lo que se asume que nuestras creencias son eminentemente lingüísticas. No obstante, si consideramos que la mente (y el cerebro) solo puede efectuar cogniciones de manera lingüística y consciente, erramos en comprender la complejidad de la comprensión humana, la cual posee más ámbitos.

¿Qué es “creer”? ¿Qué es "poseer una creencia”? Intuitivamente, nos parece que el poder expresar y comprender nuestras propias creencias nos hace dueños de ellas. Es decir, creemos que podemos dominar a voluntad nuestro sistema de creencias. Aunque esto parece cierto, los estudios de las ciencias cognitivas refutan esta perspectiva. El objetivo de este trabajo es considerar ampliamente los tipos de creencia en tanto que esta contiene representaciones mentales que intuitivamente no conocemos.

La siguiente exposición se dividirá en cuatro apartados. El primero responde a la necesidad de saber cuál es el estatus ontológico del cerebro y la mente. En otras palabras, se requiere tomar una posición filosófica sobre qué es la mente y qué es el cerebro. La segunda cuestión que debe considerarse es el carácter múltiple de la cognición en tanto hallazgos de disciplinas y ciencias cognitivas, donde se incluye la neurociencia, el psicoanálisis y la psicología budista. Esto será resuelto en el segundo y tercer apartado. A partir de esto, en la cuarta parte, determinaremos qué es una creencia considerando las premisas previas a esta definición en los apartados anteriores. Para esta última tarea del capítulo utilizaremos teorías filosóficas y psicológicas para entender este fenómeno llamado "creencia". 


\section{La inseparabilidad de la mente y el cerebro}

¿Qué relación existe entre la mente y el cerebro? En este artículo se tomará como base lo siguiente: el cerebro y la mente son dos ámbitos del mismo fenómeno ${ }^{1}$. Para fundamentar esto, utilizaré el texto I am not a brain (2017) de un autor alemán recientemente reconocido, Markus Gabriel, como objeto de críticas, de modo que sea una introducción a la aseveración principal de este apartado.

En el libro mencionado, Gabriel afirma lo siguiente: "what we call 'the mind' is really the capacity to produce an open-ended list of self-descriptions which have consequences for how humans act. Humans act in light of their self-understanding" ("lo que llamamos "mente" es en realidad la capacidad de producir una lista sin fin de descripciones propias que tiene consecuencias sobre cómo los humanos actúan. Los humanos actúan a partir del entendimiento de sí mismos", traducción nuestra) (Gabriel, 2017, p. 13). La manera en que define la mente exacerba la capacidad humana de ser autoconsciente y de decidir qué acciones tomar y qué creer a partir del lenguaje. El texto de Gabriel es, en realidad, una defensa contra el reductivismo cientificista sobre la mente, aquel que afirma que la mente humana es solo un producto de sus reacciones químicas, por lo que la voluntad y la consciencia se reducen a procesos físico-químicos. No obstante, para lograr ese objetivo, el autor en cuestión está utilizando la estrategia equivocada: parece una posición dualista en la cual la mente es inidentificable con el cerebro. Para contradecir la posición de que nuestra mente es solo reacciones químicas, arguye que la mente no depende del cerebro, sino de sí misma (menciona que la consciencia que tenemos sobre nosotros implica una creación mental a partir de otras creaciones mentales previas obtenidas a partir de la socialización). Al aseverar esto, la posición aumenta el dualismo: ninguna creencia mental es físico-química. Esta posición no está matizada.

¿Cuál es el error principal? Markus Gabriel obvia que la mente humana posee creencias inconscientes y creencias no verbales ${ }^{2}$ que son producidas de manera casi autónoma por el cerebro. En tal sentido, asumiendo esto, la mente no comprende solo verbalidad y decisión consciente, sino su contraparte asociada con procesos automáticos cerebrales: existen respuestas inmediatas a determinados estímulos y captamos la realidad de manera multimodal. Este es un ejercicio no consciente efectuado eminentemente por el cerebro.

Pese a estas objeciones, sería injusto explicar la posición de Gabriel solo por ese libro, pues en Neo-existentialism asevera lo siguiente: "the phenomena typically grouped together under this heading [the mind] are located on a spectrum ranging from the obviously physical to the non-existing" ("los fenómenos típicamente agrupados bajo este encabezado [la mente] están situados en un espectro desde lo obviamente físico hasta lo no existente", traducción nuestra) (2018, p. 14). Él está atacando, como mencioné, la postura contraria, el neuro- 
centrismo, es decir, el reduccionismo de la mente a las reacciones físico-químicas cerebrales. Para lograr este cometido, utiliza la idea de Geist, la palabra alemana traducida como "espíritu", pero que, en el texto antes citado y sus conferencias, es mencionada como las creencias de una persona basadas en la cultura propia de una época. Lamentablemente, esta crítica la llama antinaturalismo (2017), el cual no es un término apropiado para su crítica por parecer que deja la fisicalidad en el asunto de la mente. No obstante, en un debate con Searle (Gabriel \& Searle, 2018), él acepta ideas propias de la ciencia actual, como que nuestra percepción sobre la realidad es una alucinación, pero con la salvedad de que no se puede reducir la mente a ello.

Estoy de acuerdo con esta última posición de Gabriel, pero me parece que está defendiendo una postura a partir de un problema inexistente, pues es una falsa dicotomía: o el cerebro se reduce a la mente o la mente se reduce al cerebro. La posición que defiendo es la misma que Solms ha defendido en varias conferencias: "la mente y el cerebro son un mismo fenómeno visto desde la perspectiva subjetiva y objetiva, respectivamente. La mente injiere en el cerebro; el cerebro injiere en la mente".

Searle, por ejemplo, reconoce que la terminología es usualmente equívoca, pues pareciera que la mente y el cerebro fuesen conceptos que se excluyen mutuamente (2007, p.39). Según él, lo que en realidad ocurre es que nuestra mente y consciencia son un feature del comportamiento neuronal tanto como que nuestra vida mental es ontológicamente real como experiencia de la primera persona (2007, p.50). De similar opinión es Davidson, quien asume que: "Every causally interacting mental event is token-identical to some physical event" ("Cada evento mental que interactúa causalmente es idéntico a algún evento físico particular y contingente", traducción nuestra) (Yalowitz, 2014), es decir, que el cerebro determina la mente solamente en tanto que hay actividad neuronal contingente correlacionada con el acto de pensar.

Esta es una idea ya ampliamente aceptada dentro de las ciencias cognitivas. Sapolsky, por ejemplo, en su texto Behave (2017) concluye que carece de sentido el debate: "it actually makes no sense to distinguish between aspects of a behavior that are 'biological' and those that would be described as, say, 'psychological' or 'cultural'. Utterly intertwined" ("en realidad no tiene ningún sentido distinguir entre aspectos de la conducta que son 'biológicos' de aquellos que serían descritos como 'psicológicos' o 'culturales', cuando están absolutamente entrelazados”, traducción nuestra) (p. 13). Asume que una conducta no puede ser nunca entendida en tanto biológica o psicológica, sino que esta cobra sentido por la superposición de diversas causas; el fenómeno del accionar humano es resultado de diversos factores. Asimismo, Solms menciona que Freud mismo es monista de aspecto dual (Solms \& Turnbull, 2015, p. 21). Tanto el padre del psicoanálisis como el precursor del neuropsicoanálisis (Solms) están 
de acuerdo en esta posición: la mente y el cerebro son dos perspectivas del mismo fenómeno.

¿Qué es lo que se ha descubierto con estas ciencias? Propondremos cuatro argumentos que sustentan por qué mente y cerebro son perspectivas de un mismo fenómeno, en tanto que se influyen mutuamente: A) tenemos necesidades innatas no creadas culturalmente, B) las capacidades mentales pueden ser mermadas por el daño cerebral, C) las vivencias de un individuo moldean las reacciones químicas cerebrales y D) las prácticas deliberadas mentales, como la meditación, pueden cambiar el cerebro.

Respecto al primer argumento, que tenemos necesidades innatas, utilizaremos las perspectivas de Solms y Panksepp, ambos neurólogos con preparación adicional en psicoanálisis. Solms afirma (2018) que el psicoanálisis y las neurociencias están de acuerdo en que existen necesidades innatas de los seres humanos y otras especies. Tenemos un interés intrínseco por la realidad en tanto que tenemos objetivos hacia esta; tenemos instintos sexuales y buscamos una pareja para eso; necesitamos escapar del peligro (por ello, sentimos la respuesta inmediata del miedo); necesitamos destruir los objetos que nos frustran (lo cual es sentido como ira); necesitamos que nos cuiden (y sentimos pánico cuando nos separamos del cuidador) y necesitamos cuidar a los demás; finalmente, necesitamos jugar, actividad con la cual se establecen jerarquías sociales. Ninguna de estas necesidades es creada culturalmente, sino que son respuestas inmediatas ante estímulos relacionados con la supervivencia; es decir, parece que hubiese una "ética de la supervivencia", de modo que tenemos necesidades innatas para promoverla (Solms, 2015). Nos enojamos y podemos ser violentos cuando no logramos un cometido; nos asusta el peligro que asumimos como real; necesitamos que alguna figura externa nos cuide o sentiremos pánico; fomentamos los lazos de comunidad al cuidar a los demás, intentar tener sexo, o jugar. Antes de nacer, estamos programados para formar nuestra mente en función de estas necesidades.

El segundo argumento de la mente y el cerebro como aspectos de un mismo fenómeno es el hecho que el daño cerebral tiene injerencia directa en la actividad mental. Se podría poner como ejemplo las ya varias lesiones descubiertas, como la afasia de broca (incapacidad de producir lenguaje por una lesión en, precisamente, el área de broca) o la afasia de Wernicke (incapacidad de comprender completamente el lenguaje por una lesión en el área del mismo nombre), pero se podría aducir que estos ejemplos son motores o cognitivos, lo cual es distinto a la mente. Por eso, colocaremos el ejemplo ya clásico reseñado en Descarte's error (1999): el caso de Phineas Gage. Tras una explosión en su trabajo como obrero de un ferrocarril, un pedazo de hierro atravesó su cráneo. El hombre, pese a la gravedad del daño, sobrevivió. No obstante, al atravesar el lóbulo frontal, afectó un área del cerebro vinculada a la toma de decisiones. 
Después del accidente, sus compañeros y amigos lo describieron como errático e impulsivo, distinto a la ordenada personalidad que tenía; es decir, sus decisiones, las cuales son mentales, fueron afectadas por un hecho físico. De más está mencionar los perniciosos tratamientos por lobotomía a la población que le sucedieron a este evento y cómo estos cambiaron la personalidad de las personas súbita e irreparablemente.

La tercera idea que fundamenta la inseparabilidad de ambos fenómenos es que la cultura también moldea la química del cerebro (es decir, ocurre al contrario de los dos argumentos anteriores). Sapolsky (2017), por ejemplo, menciona que ciertos experimentos sociales con ratas determinan su mejora o declive mental: el experimento demostraba que el hecho de que las ratas estén en un ambiente estimulante y acogedor generaba que estas tengan mayores asociaciones mentales y mejor memoria (contaban las asociaciones dendítricas y cuán fuertes eran) (p.144). Esto demuestra cómo las vivencias suponen el detrimento o mejora de las funciones cerebrales: la imagen del mundo de una persona puede potenciar el desarrollo de funciones cerebrales físicamente rastreables. Ahora bien, se podría argüir que una rata no es un humano (pese a que la extrapolación tiene sentido por las similitudes fisiológicas). No obstante, solo he colocado un ejemplo extremo para una idea ampliamente aceptada por los psicólogos: nuestros estados mentales generan cambios fisiológicos. Esto es de tal obviedad que colocaré un fundamento simple: el pensar en una situación que reconocemos estresante genera cambios fisiológicos percibidos por el mismo sujeto. En otras palabras, con solo pensar e imaginar una circunstancia, surgen emociones, pese a que ante nuestros ojos nada esté ocurriendo. Como lo menciona Livet (2018), hay una respuesta emocional con solo pensar en una circunstancia, como si el cerebro se anticipara a esta simulándola:

When we evoke a possible future situation that differs from our present course of action and its internal dynamics, this evocation can trigger an emotional reaction that is similar to the memorized effect of the past interaction of similar external dynamics and similar past internal dynamics. (p.152)

Cuando evocamos una situación futura que difiere de nuestro presente y de sus dinámicas internas, esta evocación puede disparar reacciones emocionales similares al efecto memorizado en la situación pasada de dinámicas parecidas, externas e internas. (Traducción nuestra)

Por último, y considerando esa última idea, el cuarto argumento responde a la siguiente realidad que cada vez posee más investigaciones: es posible cambiar nuestra fisiología cerebral solo con ejercicios mentales. Este es el caso de la meditación. Aunque durante mucho tiempo no fue considerada por la psicología, lo cierto es que ahora las corrientes de mindfulness son producto de cómo el meditar - práctica hinduista y budista que consiste en intentar continuamen- 
te concentrarse en la sensorialidad o alguna idea, intentando cesar los demás pensamientos- se ha introducido a Occidente. Dicha práctica genera cambios físicos en el cerebro. Es decir, mediante el control deliberado de la mente, el cerebro cambia físicamente. Ejemplo de ello son los hallazgos de Goleman y Davidson (2017) en el cerebro de Mingyur Rinpoche, un miembro de alto rango de la jerarquía del budismo tibetano, junto a otros 12 meditadores de alto rango. No solo su percepción multimodal estaba aumentada (al medir sus ondas gamma), sino que en este Rinpoche habían detectado que su cerebro tenía la juventud de una persona de 33 años, cuando él tenía 41. De nuevo, estos son cambios físicos a través de prácticas eminentemente mentales.

\section{¿Cómo conocen los niños?}

"We are born with the ability to discover the secrets of the universe and of our own minds, and with the drive to explore and experiment until we do" ("Hemos nacido con la habilidad para descubrir los secretos del universo y los de nuestra propia mente, así como con el impulso de explorar y experimentar hasta que logremos descubrirlos", traducción nuestra) (Gopnik, Meltzkoff, \& Kuhl, 2001, p. 3). La afirmación anterior parecería descabellada, según Freud, Piaget o Wundt (Rochat, 2001), pues a principios del siglo XX aún se creía como irracionales a los niños, pequeñas personas con reacciones no conexas que actuaban aleatoriamente frente a estímulos. Los autores que usaremos en este pequeño apartado desmienten esta posición; a través de experimentaciones modernas se ha determinado que el mundo mental del niño es bastante complejo, pues tienen la capacidad a priori de reconocer patrones y la usan para entender paulatinamente la realidad.

Antes de la experiencia, según varias investigaciones (Rochat, 2001; Gómez, 2004;Gopnik, Meltzkoff, \& Kuhl, 2001), los niños pueden reconocer patrones. Por ejemplo, varios bebés son fascinados por las líneas que dividen las cosas; cuando se les graba mediante videocámara, sus ojos se dirigen a los bordes de los objetos. Paulatinamente, delimitan unidades de manera que pueden saber dónde acaba uno y comienza otro (Gopnik, Meltzkoff, \& Kuhl, 2001, p. 65). No hay una enseñanza de los patrones, sino que poseen innatamente la capacidad de delimitar representacionalmente los límites entre los objetos. Este reconocimiento de patrones como previo a la experiencia es incluso aceptado por primatólogos. Ambos, los niños y los primates, reconocen patrones en las cosas antes de usarlas (Gómez, 2004, p. 60). Es más, los bebés pueden formar categorías, diferenciar colores, diferenciar personas, diferenciar emociones arraigadas a objetos, etc. Su capacidad cognitiva es bastante amplia.

En adición, el bebé no solo observa, sino que su cognición posee dos características: representa la realidad con varios sentidos (su percepción es multidimensional) y no solo conoce una vez, sino que lo hace repetidas veces para 
generar conclusiones (investigan mediante ensayo y error). Explicaremos cada una.

La primera característica trata sobre cómo un niño puede conocer la realidad mediante varias modalidades distintas a la vez (vista, olfato, tacto, gusto y audición), incluso sin usar el lenguaje: "It has been demonstrated (...) that monkeys, apes, and human infants are all capable of cross-modal cognition without language" ("Ha sido demostrado que [...] los infantes monos, simios y humanos son capaces de cognición multimodal cruzada sin lenguaje", traducción nuestra) (Gómez, p. 2004, p. 49). Cuando el cerebro crea una conjetura sobre el mundo, el estímulo externo es amplio (ves, hueles, tocas, saboreas, oyes) $y$, de acuerdo a eso, se crea una "imagen" ${ }^{3}$ del mundo que es multisensorial. Un solo objeto es percibido de múltiples formas pese a que se vea de manera unitaria: "it has been suggested that the very notion of object arises out of the unified coordination of collected sensations that tend to cluster together" "se ha sugerido que la misma noción de objeto surge la coordinación unificada de varias sensaciones que se aúnan juntas”, traducción nuestra) (Gómez, 2004, p. 48). El objeto surge como consecuencia de una concatenación de cogniciones de diverso tipo.

Respecto a la segunda característica de la cognición infantil, la indagación mediante conjeturas y refutaciones, en algunas investigaciones se asevera que los conocimientos de los infantes se complejizan conforme tienen más experiencias. Gopnik, Meltzkoff y Kuhl (2001) hallaron que un recién nacido, pese a que puede diferenciar colores y objetos, no confía aún en esas categorías para ordenar la realidad, lo cual sí ocurre cuando cumple un año (p. 81). En otras palabras, las conjeturas son probadas repetidas veces hasta estar seguro de que es verdadera. Estos investigadores también afirman que, por ejemplo, los niños de 3 o 4 años diferencian entre lo que hay dentro de un objeto y la forma que posee: un objeto puede tener la misma forma y no ser lo mismo (p. 83). ¿Cómo ocurre la evolución de su pensamiento? Los bebés prestan atención a contraevidencia y refinan paulatinamente su conocimiento: "We seem to have a kind of explanatory drive, like our drive for food or sex" ("Parece que tenemos una especie de impulso por explicar, como el que tenemos por comer o por el sexo", traducción nuestra) (Gopnik, Meltzkoff, \& Kuhl, 2001, p. 85). Esta es una actitud científica. Y no solo ocurre con ellos: los monos bebés, como los humanos, actualizan sus representaciones mentales ante evidencia nueva más compleja conforme crecen (Gómez, 2004, p. 42). Esto es también reafirmado por hallazgos neurocientíficos: "We need to engage with the world - since all our biological appetites (including bodily needs) can only be met there" ("Necesitamos relacionarnos estrechamente con el mundo - ya que todos nuestros apetitos biológicos [incluyendo nuestras necesidades fisiológicas] únicamente pueden ser desarrollados en este—”, traducción nuestra) (Solms, 2018, p. 5). 
Ningún humano capta la realidad de manera apriorística y comprende todo inmediatamente. Como en ciencia, experimentamos e intentamos crear la mejor respuesta, tomando en cuenta la gran cantidad de información multisensorial que recibimos. Cometemos errores y revisamos nuestras inferencias para aproximarnos paulatinamente a la verdad (Gopnik, Meltzkoff, \& Kuhl, 2001, p. 16-17). Lo mismo afirma tanto Rochat —el infante es un explorador (2001, p. 89) - , como Fodor: "concept learning is a process of inductive inference; in particular, that it's a process of projecting and confirming hypotheses about what the things that the concept applies to have in common" "el concepto de aprender es un proceso de inferencia inductiva; en particular, es un proceso de proyectar y confirmar hipótesis sobre cómo el concepto aplica en situaciones con elementos en común", traducción nuestra) (Fodor, 2008, p. 132). Conocemos las características del mundo antes de usar el lenguaje comunicable entre personas; incluso podemos cambiar nuestras creencias sin ese tipo de lenguaje (el reajuste de las creencias de un bebé se hace sin lenguaje lingüístico). Conocer cómo es el mundo no es lo mismo que saber la verdad; solo hacemos nuestro mejor esfuerzo para conocer la realidad. Intentamos conocerla, pero no siempre atinamos. En ese sentido, los humanos, tanto adultos como niños, tenemos un "instinto científico": "Children are not blank tablets or unbridled appetites or even intuitive seers. Babies and young children think, observe, and reason. They consider evidence, draw conclusions, do experiments, solve problems, and search for the truth" "Los niños no son tablas en blanco ni apetitos inmanejables ni observadores intuitivos. Los bebés y los niños jóvenes piensan, observan y razonan. Consideran evidencia, arriban a conclusiones, hacen experimentos, resuelven problemas y buscan la verdad", traducción nuestra) (Gopnik, Meltzkoff, \& Kuhl, 2001, p. 13)

\section{¿Cómo funciona la mente de un adulto?}

Una vez descrita la cognición infantil, explicaremos la complejidad de la cognición adulta. Para ello, abordaré dos aspectos: los sesgos cognitivos derivados de límites perceptivos y el funcionamiento cerebro-mental respecto a las emociones. Para esto se usarán hallazgos tanto de la neurobiología como del psicoanálisis y del budismo. El objetivo es describir como nuestros la complejidad de la mente humana en tanto tenemos una biología que determina nuestras facultades en varios aspectos ${ }^{4}$.

Para ambos casos - los límites de la percepción y el funcionamiento emocional del humano - es necesario partir de esta premisa básica: la función principal de nuestro cerebro es la supervivencia. En esto están de acuerdo todos los investigadores. Entre los que utilizamos en este estudio, están Solms, Sapolsky, Dennett y Damasio, así como Padmasiri y Sangharákshita. Esta función supo- 
ne que manejamos la información que conseguimos de la realidad con el fin de sobrevivir. A priori, estamos enfocados en conseguir ese objetivo.

Entendido lo anterior, desarrollaremos el primer problema, el del sesgo cognitivo asociado a los límites de la percepción. Este, el sesgo cognitivo, es el hecho de que poseamos una creencia o efectuemos una acción sin considerar "adecuadamente" la información que la sustenta. Sobre estos, puede haber una lista muy grande; no obstante, sostengo que estos "sesgos" no son tales, sino que son límites cognitivos. Una persona puede complejizar sus conjeturas con experiencia, de manera que cometerá menos errores ahora, pero eso no significa que antes estaba sesgado o que no lo está actualmente pese a su experiencia. En realidad, los "sesgos cognitivos" no son más que el escrutinio externo de una conjetura válida, es decir, la crítica externa que utiliza la ventaja de poder tener una perspectiva más amplia. En tal sentido, solo se puede juzgar de "sesgo" a una conjetura desde una perspectiva contextualmente distinta a la inicial. Además, ese escrutinio, si bien minimiza la posibilidad del error, es de todas maneras un juicio que es él mismo "sesgado". ¿Cómo puede una persona estar segura de que no se ha equivocado en su juicio? No puede; conocer los fenómenos requiere, por esa razón, de muchas personas. Los errores son reales y las personas se equivocan; creamos conjeturas con información limitada, pues no tenemos la capacidad de la omnisciencia, pero empleamos la lógica para crearlas. En tal sentido, no hay ninguna creencia cierta en sí misma, porque no hay una evidencia absolutamente confiable, pues el conocimiento sobre la realidad es una construcción compleja que nunca para de ser construida (Sanghakárshita. 2011).

De hecho, varios autores ya han dejado aclarado la limitación de nuestra cognición. Analicemos las ideas de dos investigadores en concreto: Antonio Damasio y Daniel Dennett. El primero, neurocientífico, afirma (2010) que el humano crea constantemente mapas; es decir, halla patrones en la realidad, de manera que la entiende de acuerdo a estos (esto debería ser evidente ya desde Davidson, quien asevera que no hay cognición pura, pues el mismo hecho de conocer implica aplicar un esquema a la data empírica). En tal sentido, entendemos la realidad mediante ese patrón, por lo que no observamos todas las posibilidades que diferentes marcos conceptuales nos pueden ofrecer al analizar un fenómeno. Nuestro cerebro crea, con un marco conceptual, y los datos que tiene disponibles, suposiciones. Estas tienen, siempre, data limitada que nuestro cerebro completa: toma información anterior que sea plausible y la acomoda para que tenga sentido. De la misma opinión son Bennett y Hacker: "the brain has experiences, believes things, interprets clues on the basis of information available to it, and makes guesses" "el cerebro tiene experiencia, cree cosas, interpreta pistas a partir de la información disponible y [luego] hace conjeturas", traducción nuestra) (2003, pág. 68). Estas conjeturas, después, se convierten en 
creencias que forman parte de nuestro esquema mental que nos guiarán para analizar situaciones similares.

Hasta este punto, hemos descrito dos características interesantes del cerebro: A) no podemos usar toda la data disponible y B) utilizamos modelos para entender la realidad de manera limitada. Además de ello, si seguimos a Dennett, es evidente que el entendimiento humano es más limitado aún: no solo usamos patrones para limitar las posibilidades, sino que usamos patrones episódicos. Como él mismo menciona: "the information (...) does not have to be sent somewhere else to be rediscriminated by some 'master discriminator" ("la información no tiene que ser enviada a otro lado para ser discernida nuevamente por un 'discernidor maestro”, traducción nuestra) (Dennett 1991, pp. 112-113). No hay ningún "yo" que considere todas nuestras creencias o nuestros principios y, de acuerdo con ello, juzgue la mejor acción posible. En realidad, la mente crea multiple drafts (Dennett, 1991), es decir, varias conjeturas contextuales que no necesariamente están conectadas; hallamos patrones en la realidad que responden a circunstancias particulares. Nuestras reacciones no son controladas, sino que repiten patrones (siguen las rutas ya "cableadas" por el cerebro) de acuerdo con la circunstancia (es episódico).

Formados los patrones circunstanciales arriba descritos (conjeturas episódicas a partir de data limitada), entendemos la realidad a partir de ellos. Dennett, en Consciousness explained (1991), explica esto: tenemos una especie de "cableado duro" a partir de la identificación de patrones y, a partir de esto, creamos una forma de estereotipos que confrontamos continuamente con la realidad. Usualmente, los reforzamos. Todo lo que entendamos ya está limitado por patrones inconscientes que hemos creado desde niños, sobre todas en nuestras primeras conjeturas, las que no hemos refutado. En palabras de Sanghakárshita (2011), tenemos la costumbre de tener un cerebro reactivo en lugar de uno creativo. Esto ocurre precisamente porque utilizamos el mismo esquema mental para distintas situaciones, de manera que no tengamos que hacer perpetuamente conjeturas. Es una especie de economía mental. Detallaremos este asunto hacia el final de este apartado.

Como bien lo menciona repetidamente el psicoanalista y neurocientífico Solms, el ser humano tiene la función de conservación personal: la percepción del hombre se emplea para asegurar que sobrevivirá. Esta función es aún más clara en las reacciones emocionales. En esta segunda parte del apartado, abordaremos el asunto de los límites cognitivos asociados a estas.

Las emociones son "complex, largely automated programs of actions concocted by evolution" ("programas de acciones automatizados y complejos que se crearon a partir de la evolución", traducción nuestra) (Damasio, 2010, p. 95). Es decir, una emoción es una configuración que produce una acción automatizada que cumple una función para que el individuo se adapte al medio, 
con tal de que se asegure su supervivencia. ¿Cuál es la evidencia de esto? Desde una perspectiva subjetiva, esta definición es ampliamente comprobable: hay acciones particulares que deseamos hacer cuando nos sentimos tristes, felices, enojados, disgustados, sorprendidos o asustados ${ }^{5}$, y todas tienen como función conservarnos. Esto supone la existencia de reacciones químicas determinadas que suponen todo un plan de reacción corporal ante determinados estímulos.

En adición, esto puede ser comprobado de forma objetiva: Sapolsky otorga pruebas claras de esta respuesta automatizada. Menciona que existe una relación intrínseca entre la corteza prefrontal y el sistema límbico. En este último, está tanto la amígdala como el hipotálamo, el primero relacionado a funciones de control de ira y miedo, y el segundo relacionado con nuestras reacciones autónomas (sistema simpático y parasimpático). Estos son órganos altamente emocionales, por lo que probar que hay una relación intrínseca con la corteza frontal, encargada del pensamiento, implica que el procesamiento de información es, a la vez, emocional e intelectual; no hay dicotomía. De la misma opinión es Damasio quien asevera que la racionalidad surge de la regulación biológica de las emociones, a la vez que opera junto a ella: la racionalidad surge tanto del cerebro "viejo", el emotivo, y del "nuevo", la corteza prefrontal que nos otorgó la capacidad de pensar lingüística y complejamente (1994, p. 128).

Un ejemplo del funcionamiento de la respuesta automática ante un estímulo es la división nosotros/ellos: "By age three to four, kids already group people by race and gender, have more negative views of such Thems, and perceive other-race faces as being angrier than same-race faces" ("Para la edad de tres o cuatro, los niños ya agrupan a las personas por raza y género, tienen más visiones negativas de los 'otros' y los perciben como con gestos más negativos que aquellos que tienen su misma raza”, traducción nuestra) (Sapolsky, 2017, p. 405). Sapolsky aumenta además lo siguiente: "The automaticity of Us/Theming is shown by the speed of the amygdala and insula in making such dichotomies - the brain weighing in affectively precedes conscious awareness, or there never is conscious awareness, as with subliminal stimuli" ("La automaticidad de hacer la dicotomía Nosotros/Ustedes está mostrada por la velocidad en que la amígdala y la ínsula hace esas dicotomías - la intervención afectiva del cerebro precede la consciencia o incluso ni siquiera ocurre dicha toma de consciencia, como con el estímulo subliminal", traducción nuestra) (Sapolsky, 2017, p. 414). A partir de esto, enfatizamos la inmediatez de la respuesta: son dicotomías útiles para protegernos de los peligros, es decir, una especie de programación diseñada por la evolución que intenta garantizar la supervivencia. Claro está, el otro aspecto de este fenómeno supone el empleo de estas respuestas como fomento de la integración: la división nosotros/ustedes también implica que hay personas frente a las que puedo sentir alegría o filiación. No obstante, esto depende mucho de las experiencias personales de cada individuo: "As a really interesting contingent effect, oxytocin enhances charitability - but only in 
people who are already so. This mirrors testosterone's only raising aggression in aggression-prone people" "Como un realmente interesante efecto contingente, la oxitacina aumenta la capacidad de ser dadivoso - pero solo con personas con quien uno ya lo es-. Esto es parecido a [los hallazgos donde] la testosterona aumenta la agresión en quien está predispuesto a", traducción nuestra) (Sapolsky, 2017, p.118). En tal sentido, las respuestas emocionales dependen también de nuestras vivencias, incluso hormonalmente. De la misma opinión es Damasio (2010), quien afirma que debido a que las emociones son disposiciones genéticas, el desarrollo de una u otra respuesta emocional es reafirmada por experiencias distintas. La reacción emocional ante determinado estímulo es una respuesta adaptativa particular que estaba ya supuesta en nuestra carga genética, pero como posibilidad.

Podríamos aumentar acá, para agregar complejidad al asunto, que las emociones tienen un detonante cognitivo que no parece obvio para nosotros, quienes adoptamos una cultura occidental. Por ejemplo, el miedo solo surge cuando una persona tiene deseos fuertes sobre un objeto o apego hacia este: aquel se defiende contra posible pérdida del objeto. Lo anterior puede ocurrir tanto con objetos no animados (una casa, dinero, un objeto con valor afectivo) o seres vivos (personas que queremos, animales que queremos). Lo mismo ocurrirá respecto a uno: nosotros somos uno de los objetos que más valoramos y, por lo tanto, la ansiedad surge cuando pensamos que podemos perder integridad (Padmasiri, 1979, p. 46) (por eso, enfatizamos tanto que nuestras emociones son reacciones ante la posibilidad de no sobrevivir). Esto puede ser extremadamente difícil de aceptar, pues usualmente aceptamos la idea a priori de que nuestras emociones son espontáneas e incontrolables, cuando estas actúan a través de representaciones (Padmasiri, 1979, p. 51). Solo si aceptamos, por ejemplo, que queremos continuar con la existencia y que la existencia es deseable, podemos sentir miedo. Evidentemente, esta aseveración puede resultar escalofriante, pero el objetivo de esta reflexión, como asevera Padmasiri (1979), es que es necesario saber los orígenes de las emociones para poder manejarlas mejor. Esa es, por ejemplo, la intención de la liberación de la doctrina budista.

Volvamos a la descripción de la mente humana. Según esta explicación, ha de quedar claro que estas reacciones automáticas, llamadas emociones, tienen la función de supervivencia inmediata; para eso sirven. No obstante, este fenómeno posibilita el hecho de ser conscientes de la emoción, lo cual hace posible la representación mental. Esto último configura otro fenómeno distinto al de la emoción, los sentimientos. La percepción de la emoción, el sentimiento, imbrica varios fenómenos. Como Damasio lo define, los sentimientos son la percepción compleja de sentir las emociones (1999, 2010). En tal sentido, ocurrida la emoción, el humano percibe ilusoriamente que la emoción forma parte de sí. No obstante, son fenómenos distintos: "While emotions are actions accompanied by ideas and certain modes of thinking, emotional feelings are 
mostly perceptions of what our bodies do during the emoting, along with perceptions of our state of mind during that same period of time" ("Mientras que las emociones son acciones acompañadas de ideas y ciertos modos de pensar, los sentimientos de las emociones son percepciones acerca de qué es lo que nuestros cuerpos hacen al emocionarnos, junto con percepciones de nuestro estado mental durante el mismo periodo del tiempo", traducción nuestra) (Damasio, 2010, p. 88$)^{6}$. Esta demarcación entre ambos conceptos debe quedar clara, pues es necesario considerar la separación entre el fenómeno y la cognición del fenómeno. Este es un principio budista e hinduista desde hace miles de años y es central para entender cómo las reacciones emocionales y los sentimientos son racionales. Mingyur Rinpoche (2017), por ejemplo, nos explica que la mente reactiva, aquella que se guía por las emociones, es llamada por la tradición como "mente de mono". Esta cosmovisión no asume, como lo haría un occidental, que esta condición es deleznable o no humana; al contrario, sabe que es parte de nuestra configuración cerebral e intenta integrarla a nuestra cognición. La "mente de mono" asume que las programaciones emocionales son reales y que debe estar buscando continuamente cómo sobrevivir, por lo que el estado de alerta es necesario siempre. Por esa razón este rinpoche menciona que una forma astuta de calmarlo es darle tareas, como atender a un objeto y concentrarse en ello: ese adiestramiento es llamado meditación.

Ahora bien, esta representación mental de la emoción —el sentimiento-, lo repito, tiene el objetivo de cumplir la función de supervivencia. Como lo menciona Damasio, cumplen el rol de seguir el imperativo homeostático (2018, p. 87); no son un lujo, sino que son un especie de guía interna que comunica un estado (de todas maneras mediado por la interpretación) sobre la realidad (Damasio, 1994, p. xv). Además, es un reporte en tiempo real del estado de nuestro cuerpo de manera multidimensional (p. 76). Esto último es bastante interesante: la información que asociamos a las emociones es una representación mental que puede ser visual, olfativa, sensorial, gustativa, auditiva o lingüística (y comúnmente concurren varias a la vez).

El asunto es complejo: la percepción de una representación mental crea emociones que la refuerzan y, a partir de ello, se crean más representaciones que pueden crear más emociones iguales o distintas a la primera. Hay una especie de multitiriggering de respuestas a través de una representación mental7. Un ejemplo de esta complejidad es lo aseverado por Elster "If I know that I'm experiencing a certain emotion, that cognition may serve to trigger further emotions, or meta-emotions" "Si yo sé que estoy experimentando cierta emoción, esa cognición puede servir para disparar más emociones, o meta-emociones", traducción nuestra) (1999. p. 255). Uno podría sentir, por ejemplo, vergüenza por sentirse triste, culpa por sentir afecto, etc. Sin reparar en ello, los humanos podemos sentir emociones al tener la representación mental del fenómeno de cierta emoción: las emociones desencadenan un complejo de creencias que 
crean más emociones que crean más creencias, etc. Esto ciertamente es entendido por Elster como capacidades defectuosas para la toma de decisiones (1999, p. 293). No obstante, este es un malentendido. Las decisiones se toman con las emociones; sin ellas, no solo no nos defenderíamos del peligro, sino que nos costaría decidir qué es beneficioso para nosotros, pues tampoco tendríamos sentimientos que promovieran integración.

Además de la complejidad que he presentado sobre cognición, emociones y sentimientos, involucraremos un fenómeno más, las necesidades innatas del humano; basados en las perspectivas de Solms y Panksepp, ambos neurólogos con preparación adicional en psicoanálisis. El primero (2018) afirma que el psicoanálisis y las neurociencias están de acuerdo en que existen necesidades innatas en humanos y en otras especies. Tenemos un interés intrínseco por estar involucrado con nuestro medio (y tenemos objetivos dentro de este); tenemos instintos sexuales (buscamos una pareja para eso); necesitamos escapar del peligro (por ello, sentimos la respuesta inmediata del miedo); necesitamos destruir los objetos que nos frustran (lo cual es sentido como ira); necesitamos que nos cuiden (y sentimos pánico cuando nos separamos del cuidador) y necesitamos cuidar a los demás; finalmente, necesitamos jugar, actividad con la cual se establecen jerarquías sociales.

Aparte de la complejidad antes mencionada (las emociones) es imperativo involucrar estas siete necesidades, pues están ligadas, como es evidente, a la supervivencia. Son, como las emociones, una especie de programas innatos de los humanos, pero son más complejos, no inmediatos como las emociones:

The human infant is not a blank slate; like all other species, we are born with innate needs (...) The main task of mental development is to learn how to meet our needs in the world (...) Most of our action plans (i.e. ways of meeting our needs) are executed unconsciously. (Solms, 2018, p. 6)

El infante humano no es una tabula rasa; como las demás especies hemos nacido con necesidades innatas (...) el objetivo principal del desarrollo mental es aprender como satisfacer nuestras necesidades en el mundo (...) La mayoría de nuestros planes de acción (es decir, maneras de satisfacer nuestras necesidades) son ejecutadas inconscientemente. (Traducción nuestra)

En esta cita, Solms explica las dos funciones esenciales de nuestro cerebro y cómo lo ejecuta. Básicamente, tenemos necesidades que deseamos realizar, por lo que usamos nuestra mente; no obstante, al ser demasiada información la que tenemos que utilizar para realizar lo que deseamos, la mayoría de conocimientos son utilizados de manera inconsciente. El problema es que ese conocimiento inconsciente se resiste a cambiar: una vez que asumimos como realidad una interpretación nos resistimos a cambiarla debido a que ha sido automatizada (Solms, 2018, p. 6). En este punto, he de explicar la complejidad de la cognición 
a partir de un principio ya mencionado en este apartado y muy criticado cuando Freud lo acuñó, pero ahora ampliamente aceptado ${ }^{8}$, el inconsciente.

Las representaciones mentales, los sentimientos y las necesidades básicas son una gran cantidad de información, entre data nueva de la realidad y programas innatos. Son demasiados datos mezclados a la vez: ¿Qué puede hacer el cerebro para asegurar la supervivencia? Automatiza procesos: esto es el inconsciente. Simplemente, significa que no reflexionamos sobre cada acción, sino que automáticamente reaccionamos. No tiene un significado esotérico; solo es la oposición a la reflexión creativa: "At a nonconscious level, networks in the prefrontal cortex automatically and involuntarily respond to signals arising from the processing of the above images" ("En un nivel inconsciente, las conexiones de la corteza prefrontal automática e involuntariamente responden a señales que provienen de procesar imágenes”, traducción nuestra) (Damasio, 1994, p. 136). En esto, todos están de acuerdo. No obstante, los psicoanalistas asumen que hay creencias más complejas automatizadas, es decir, que actuamos como actuamos a partir de asunciones complejas de la realidad, las cuales forman nuestras motivaciones. No hemos planificado nuestra vida, sino que actuamos en un marco determinado de creencias que prefiguran nuestra intención. Eso es el inconsciente dinámico.

Colocaré un ejemplo para ilustrar este punto. Para empezar, explicaremos uno que podría ser considerado controversial:

[In young rats even aversive things are reinforcing in Mom's presence, even if Mom is the source of the aversive stimuli. As Sullivan and colleagues wrote, "attachment [by such an infant] to the caretaker has evolved to ensure that the infant forms a bond to that caregiver regardless of the quality of care received." Any kind of mother in a storm. (Sapolsky, 2017, p. 199)

En ratas jóvenes incluso cosas aversivas refuerzan la presencia materna, incluso si la madre es la fuente de tal estímulo aversivo. Como Sullivan y colegas escribieron, "el apego del infante a su cuidador ha evolucionado para asegurar que este forme un lazo con su cuidador pese a la calidad de cuidado recibido". (Traducción nuestra)

En este ejemplo, queda claro que las personas cumplen sus necesidades complejamente: la necesidad de ser cuidado puede obligar a una persona a necesitar a una persona a revivir situaciones en las cuales puede sentir emociones negativas (ansiedad, miedo, enojo, etc.), porque concibe, a través de lo que ha vivido, que esa es la forma en la cual puede satisfacer su necesidad innata de protección y pertenencia. Su representación mental respecto a la satisfacción en una relación íntima supone sentir emociones de estrés; como no ha reflexionado sobre esa idea y solo actúa de acuerdo con ella, esa creencia es inconsciente. Bien podría ocurrir lo contrario, que una persona busque intimidad sin buscar 
dolor, como el caso del estudiado apego seguro; de todas formas, es inconsciente. Finalizaremos esta digresión con una cita de Fodor, quien se pregunta si la epistemología ha logrado entender la profundidad de los hallazgos de, en sus palabras, "revolución freudiana":

I sometimes wonder whether our epistemology has quite caught up with the Freudian revolution in psychology. There is every sort of evidence that a great deal of the reasoning involved in the causal fixation of quotidian perceptual beliefs is unconscious and hence unavailable for report by the reasoner. (Fodor, 2008. p. 193)

A veces me pregunto si la epistemología ha alcanzado la revolución freudiana en psicología. Hay todo tipo de evidencia de que una gran cantidad de razonamiento involucrada en la fijación causal de las creencias respecto a las percepciones cotidianas es inconsciente y, por lo tanto, no disponible para el reporte del razonador. (Traducción nuestra)

Pese a lo anterior descrito, me gustaría enfatizar lo siguiente: esta es una configuración humana. Nuestras emociones y necesidades innatas no ocurren igual en todos y, si suceden de esta forma en la mayoría, esto es solo fruto de una configuración biológica, la actual. No es una naturaleza humana perpetua, sino una sujeta al cambio. Se puede afirmar con Sangharákshita (2011) y con la escuela Mahayana que estas no son realidades inamovibles. De hecho, las emociones, como reacciones psicofísicas, son circunstanciales; la ciencia ha logrado obtener rasgos universales, pero estos tienen una genealogía, en este caso evolutiva. La configuración cerebro-mental humana se modifica cada generación. Por tanto, los rasgos psicológicos son también circunstanciales, en tanto forman parte de la evolución, es decir, de la adaptabilidad al medio. Es posible "recablear" las creencias, pues nuestro cerebro es plástico, es decir, modificable. Dennett fundamenta esto a partir del Efecto Baldwin (en biología evolutiva, se asevera que sobrevive el más apto en tanto puede aprender nuevos comportamientos que lo ayuden en esta tarea). Sin embargo, cambiar las creencias es lo difícil: los patrones aprendidos inicialmente se reestructuran con dificultad, pues son mediados por el inconsciente.

\section{¿Qué es una “creencia”? Conjeturas filosóficas y psicológicas}

Después de haber comprendido la complejidad del funcionamiento de la mente humana, además de establecer su estatus ontológico en la primera parte, podemos definir, a partir de una confrontación de la filosofía con la psicología, qué significa que un humano posea una creencia. Todo el trabajo anterior se explica por el objetivo final: determinar que las creencias están dentro de varias dimensiones posibles de cognición. 
Para este objetivo, propondremos una definición de este término y la defenderemos en este apartado: una creencia es información proposicional que un humano ${ }^{9}$ asume como real y que no es exclusivamente lingüística ${ }^{10}$, además de poder ser consciente o inconsciente. Este concepto se discutirá a través de tres autores de distinta perspectiva: Davidson, Fodor y Dennett. Pese a que tradicionalmente se les ha considerado como pertenecientes a distintas orientaciones (Schwitzgebel, 2015), observamos coherencia entre ellos en lugar de discordancia. A partir de los aportes de las diversas ciencias cognitivas que hemos explorado en los anteriores apartados, consideramos que es estéril delimitar entre interpretacionismo, representacionalismo y funcionalismo. Estos enfoques comparten bastantes ideas como para desarrollarlos aparte. Como acertadamente asevera Dennett desde Consciousness explained (1991) hasta Sweet dreams (2005), la filosofía de la mente debe tomar en cuenta las ciencias cognitivas para crear teoría: esta otorga puntos en común entre autores distintos.

Respecto a la definición sostenida, que una creencia es información no únicamente lingüística que el humano asume como verdad consciente o inconscientemente, debe explicarse tres puntos. El primero es la definición de información proposicional asumida como verdad y cómo esta puede no ser lingüística ${ }^{11}$. Establecido que entendemos la realidad proposicional y multimodalmente, explicaremos (de nuevo) cómo un humano puede poseer creencias inconscientes. No redundaremos en el asunto de las emociones, ya explicado en el anterior apartado, pero a partir de lo explicado reformularemos el concepto que se estableció al comienzo.

Para resolver la primera interrogante, podremos definir "información proposicional" de manera parecida a "actitud proposicional", es decir, data que supone comprensión mediante proposiciones. El cerebro procesa la información utilizando "conjunciones" entre una cosa y un atributo de ella. Ejemplos de esto son los siguientes: "la casa es verde", "ese abogado es honesto", etc. Explicado así, parece que una actitud proposicional es eminentemente lingüística. De hecho, uno de los autores que he mencionado, Davidson, afirma que el atributo principal de una creencia es que es lingüísticamente cognoscible (Hernández, 1994). Este es un grave problema en su trabajo: asume que las actitudes proposicionales son esencialmente lingüísticas, lo que lo conduce a afirmar que los animales carecen de actitudes proposicionales, por no poder integrar nuevos conceptos sobre una red de creencias (Davidson, 2001, p. 137). El considerar que una persona posea actitudes proposicionales únicamente por tener lenguaje es un límite para su teoría ${ }^{12}$, no solo porque no puede probar que todas nuestras creencias pueden ser traducidas a lenguaje comunicable, sino porque es evidente que los humanos empleamos mucha información de manera proposicional a través de varios estímulos sensoriales, pese a que no hayan logrado ser expresados de manera lingüística. 
En efecto, sí existen creencias que a priori no podemos comunicarlas a otro humano; poseemos un know how previo a poder expresar con palabras lo que uno sabe lingüísticamente (Fodor, 1992). Por ejemplo, uno puede saber cómo conducir una bicicleta perfectamente: saber cuándo y cómo mover las piernas, cómo calcular los movimientos para no chocar, etc. Todo eso supone flujo de información internamente, pues nuestro sistema nervioso controla el uso de las extremidades. Sin embargo, ¿es usual que podamos, de inmediato, explicar cómo realizar este ejercicio tan detalladamente como lo ejecutamos? Como obviamente la respuesta es no, debemos considerar que el cerebro puede poseer y utilizar información sin haberla comprendido mediante lenguaje ${ }^{13}$. En tal sentido, Fodor (1992) afirma que existe un "lenguaje de la mente" llamado mentalés ${ }^{14}$. En otras palabras, el cerebro recibe y transmite información en alguna especie de código para que pueda ser utilizada por el humano, pero sin que sea pensado a través de lenguaje. Este "idioma” no es lingüístico sensu stricto, pero posee la característica de comunicación interna (que también asumimos que se efectúa mediante proposiciones) que logra que alguien pueda realizar una acción a través de conocer. ¿Cómo sabemos que esto existe? Como mencioné, el humano actúa como si supiera lo que no puede comunicar lingüísticamente; eso es la "actitud proposicional". Fodor está aseverando que el mentalés es un lenguaje que acepta radicalmente que el humano guía su accionar, aunque no esté consciente de ello, por medio de información a la cual podemos llamar actitudes proposicionales (Fodor, 1992 y 2008).

Hemos de recalcar, de todas formas, que este es un "lenguaje" en tanto tiene las siguientes características recapituladas por Rescorla (2019): el mentalés solo supone la representación mental de la realidad de manera semántica con la característica de tener valor de verdad. Como es una recopilación de información, la imaginamos como proposiciones. Por ejemplo, el percibir con los ojos que un árbol es marrón supone esa proposición como información ("el árbol es marrón”) pese a no haber sido formulada lingüísticamente por el agente; la definición se hace a partir de tener la lógica como característica principal en la recolección de información. A su vez, he de recalcar el carácter hipotético de esta teoría: Fodor está razonando de manera abductiva: tenemos razones para asumir que la Hipótesis del Lenguaje de la Mente es real porque hay vestigios en las diversas teorías psicológicas actuales (Rescorla, 2019).

Ahora bien, Dennett no aceptaría por completo lo que menciona Fodor: ¿cómo sabemos que esta comunicación interna dentro del cerebro está estructurada lingüísticamente? (Dennett, 1969, p. 16). Esta objeción es válida, pero como antes mencioné, la intención de Fodor es afirmar que la transmisión de data no puede ocurrir de otra forma que con la proposición. El lenguaje es básicamente comunicación (Sapolsky, 2011) y no es necesario especificar si tiene un sentido lingüístico sensu stricto: el hecho de que una persona actúe supone transmisión de información interna que es data proposicional. Si esta persona 
no puede comunicarla lingüísticamente, ella ha recibido y empleado la información de manera no-lingüística. Esta manera no-lingüística de comunicación es lo que Fodor llama mentalés.

Dennett no apoya directamente esta tesis —además de la anterior razónporque la teoría de Fodor aún supone un centro de operaciones cerebral de representaciones mentales ${ }^{15}$, lo cual es ampliamente debatido en Consciousness explained. Es claro que, a partir del hecho de la inexistencia de un centro para la conciencia, Dennett apuesta por el hecho de que no tenemos un conocimiento lingüístico de varias de nuestras creencias (en realidad, considera que el cerebro crea conjeturas sobre las cuales no reflexionamos). En tal sentido, ni siquiera somos conscientes de lo que nuestro cerebro acepta como verdad, pero actuamos de acuerdo con ello. Como él mismo afirma, nuestro cerebro puede ser engañado por una ilusión óptica y creer directamente que no es una ilusión, sino realidad (más aún, ¿̇no es la realidad una ilusión que genera nuestro cerebro? ${ }^{16}$ ). La teoría de Fodor se mantiene, no obstante, pues es posible creer en diversidad de proposiciones sin reflexión ni un "centro" que medie cuáles se aceptan o no.

Por último, después de establecer que las creencias del humano son representaciones mentales de información tanto lingüística como no-lingüística, es necesario abordar el asunto de las creencias inconscientes. De hecho, esa idea se condice con todo lo expresado anteriormente, pues las creencias no-lingüísticas son pensadas, pero no reflexionadas, por lo que son susceptibles de no ser conscientes. Explicaremos este problema a continuación.

Se distinguirá, como se hizo en el tercer apartado, a los procesos inconscientes cognitivos de los procesos inconscientes dinámicos (Eagle, 2018). Inconscientemente percibimos varios aspectos de la realidad: no somos conscientes de cómo el cerebro percibe la realidad (por eso, como lo menciona Dennett, las ilusiones ópticas nos engañan). Lo único que sabemos es que el cerebro ya estaba preparado para comprender, desde elementos como altura, largo y ancho, tareas tan complejas como cómo caminar sin pensarlo. Hasta ese asunto, todos estarían de acuerdo, pero ¿es cierto que tenemos deseos y motivaciones (que suponen una creencia compleja) de los cuales no somos conscientes? Respecto al primer asunto, incluso Dennett y Fodor están de acuerdo en que la mayoría de procesos mentales son inconscientes (Eagle, 2018), pues no los determinamos nosotros mismos; la palabra "inconsciente" la utilizamos para afirmar que no somos conscientes de todos los procesos mentales que ocurren en nuestro cerebro. El Multiple Draft Theory de Dennett asegura precisamente que nuestra cognición no posee un centro donde logremos conocer lingüísticamente toda la información que percibe el cerebro. No reflexionamos sobre todo lo que percibimos del mundo. Las percepciones son transformadas en información que creamos a través de conjeturas que no controlamos; posteriormente, mediante reflexión, sabemos que reaccionamos de tal u otra manera por las conjeturas 
previas. Se puede entender que eso ocurra con el modo de entender la realidad física, por ejemplo, pero el problema surge cuando intentamos demostrar que existe un "inconsciente dinámico", es decir, creencias que supongan intenciones, deseos o motivos y que sean, a la vez, inconscientes.

Probablemente, el problema de aceptar esto último surja a partir de la formulación freudiana que hace pensar que el inconsciente es un alter-ego que nos domina en función de sus propios deseos. Evidentemente, una teoría así estaría enfocada en demostrar que hay un marionetista que está detrás nuestro, intentando controlarnos continuamente. Utilizar ese enfoque puede generar lo siguiente: " the costs of such presumed depth include hyperbole (...)[are], lack of evidence, and above all, lack of applicability, and enormous distance between the account and the lives of real individuals" ("el costo de esa presunta hipérbole profunda son la falta de evidencia y, sobre todo, la falta de aplicabilidad, y una enorme distancia entre esa teoría y la vida de individuos reales", traducción nuestra) (Eagle, 2018, p. 29). Pensar que, a priori, el inconsciente es una entidad aparte ha llevado a teóricos como Popper en Miseria del historicismo (1944) a mencionar que el psicoanálisis solamente está orientado a buscar ad hoc interpretaciones que tan solo refuercen su teoría sin fijarse en la evidencia real.

El psicoanálisis contemporáneo no ha sido acrítico a Freud. Una interpretación que traduce la teoría freudiana en términos más fáciles es la de Wakefield: él asegura que la esencia de los estados mentales es la representacionalidad. Siguiendo esta exégesis, Freud estaba aseverando que la mente tenía como función representar la realidad. Esta representación a través de conjeturas no solo podía ser consciente sino también inconsciente: "As Wakefield (...) shows, Freud's implicit reasoning is that if, like conscious states, nonconscious representational brain states can generate intentionality and intelligent behavior, then one must conclude that it is representational structure, not consciousness, that is the essence of the mental" "Como lo muestra Wakefield, el razonamiento implícito de Freud es que si, como los estados conscientes, los estados representacionales inconscientes del cerebro pueden generar intencionalidad y comportamiento inteligente, entonces uno debe concluir que la estructura representacional, no la consciencia, es la esencia de lo mental", traducción nuestra) (Eagle, 2018, p. 9). Nuestro cerebro representa la realidad, seamos conscientes de ello o no. Esto podría ser apoyado, en parte, por Davidson: "Most of our desires, for example, depend on our beliefs. We would not want to make money unless we believed it would put us in a position to obtain things we need or value" ("La mayoría de nuestros deseos, por ejemplo, dependen de nuestras creencias. No querríamos hacer dinero al menos que creamos que nos pondría en una posición donde podamos obtener lo que necesitamos o valoramos", traducción nuestra) (1995a, p. 16). Los deseos pueden ser inconscientes, aumentamos, pues corresponden a una representación compleja de la realidad que no siempre está explicitada lingüísticamente. 
Al comprender que así se entiende la mente, como una que principalmente representa la realidad, se puede entender cómo a partir de eso uno puede crear creencias más complejas que también forman parte de nuestras intenciones. Justamente por eso el psicoanálisis contemporáneo juzga de importantes las creencias y expectativas que se crean a partir de las primeras (Eagle, 2018, p. 31-32). Uno crea representaciones de la realidad que se convierten en creencias complejas que, finalmente, son expectativas sobre cómo va a ser la realidad. No obstante, todo este complejo de creencias está mezclado con emociones y sentimientos respecto a una u otra interpretación sobre el mundo. Por lo tanto, lo que ocurre con nuestra mente es que tenemos creencias implícitas sobre cómo funciona y es la realidad, y a partir de ellas poseemos intenciones y motivaciones. La comprensión del mundo prefigura nuestras motivaciones.

\section{Conclusión}

En conclusión, a partir de todo lo expuesto, hemos de recalcar que las creencias no pueden simplemente ser aquellas proposiciones que reconozcamos lingüísticamente como propias. Estas son representaciones mentales multimodales que detallan la realidad proposicionalmente. Estas pueden ser conocidas lingüísticamente por el sujeto que las posee, pero eso no supone que todas sean cognoscibles del mismo modo. Uno puede poseer distintos tipos de representación mental y puede no expresarlos en palabras de inmediato, pese a actuar directamente de acuerdo con ellos. La mente humana es compleja en tanto que nuestra capacidad cognitiva es diversa y múltiple, a la vez que económica y restringida.

Según el análisis hecho, se han hallado tres tipos de creencias aparte de la consciente y lingüística. La primera es la creencia inconsciente. Contrario a lo que se pueda entender de la palabra, no es una creencia ex nibilo que no podemos conocer, sino que fue consciente en algún momento de nuestra vida pero se automatizó como parte de las reacciones inmediatas que nuestro cerebro utiliza para operar en un mundo con tanto flujo de información constante. En tal sentido, cuentan tantas creencias sobre cómo efectuar un trabajo mecánico, hasta motivaciones personales que no tenemos presentes en la consciencia continuamente. Nuestro cerebro crea reacciones automáticas ante la presencia de determinadas circunstancias. El segundo tipo de creencia es la no lingüística. Esta se podría confundir con la anterior, pues usualmente no somos conscientes lingüísticamente de cómo los olores o las imágenes, etc. pueden afectar nuestra perspectiva sobre la realidad. No obstante, conforme a nuestras experiencias, tenemos información sobre cómo la realidad se estructura: hacemos diferencias y trazamos límites cognitivos para comprender la realidad, pero ello no es usualmente reflexionado en palabras. Por último, la tercera forma de creencia es una del tipo biológico: las emociones. Estas, contario a la creencia popular, son una especie de programas de acción innatos. De acuerdo con determinadas 
circunstancias (lo cual también depende de las primeras impresiones), tenemos la capacidad de sentir en alguna parte del cuerpo emociones que nos impelen a acciones determinadas. Por ejemplo, el miedo nos puede impulsar a atacar o huir. Entendido de esa forma, el flujo de información proposicional existe y la acción es efectuada a partir de esa data. Justamente como es al fin y al cabo información proposicional, uno puede llegar a reflexionar sobre ella. De esta forma, es posible no reaccionar conforme a las emociones y observarlas, tal como la práctica meditativa y terapéutica lo sugiere.

Queda claro, entonces, que reducir el término "creencia" a solo aquello que podemos comprender lingüísticamente, y de lo cual podemos tener consciencia inmediata, es un absurdo. Todas estas disciplinas de la mente han demostrado las creencias de un individuo forman parte de la complejidad de la mente humana.

\section{Notas}

1 Quintanilla (2019) lo describe como monismo de la sustancia y dualismo de las propiedades.

2 Esto será desarrollado con mayor detalle en los apartados siguientes; en este queda decir que la mente automatiza procesos simples y complejos, por lo que varias de nuestras creencias son reacciones automáticas llamadas inconscientes; y que captamos la realidad de manera multimodal, por lo que la forma en que representamos la realidad no es únicamente lingüística, sino visual, olfativa, auditiva, táctil e incluso en cuanto a sabores

3 Colocamos "imagen" entre comillas, pues esta palabra evoca la idea de un solo sentido, la visión. En realidad, me estoy refiriendo a que son conjeturas multisensoriales. Esto es mejor desarrollado en el cuarto apartado, donde explicamos la hipótesis del Lenguaje de la Mente postulado por Fodor.

4 No abogo por un determinismo biológico. Esto se explica mejor en el primer apartado donde explico cómo el cerebro y la mente son dos perspectivas del mismo fenómeno.

5 Estas nos son emociones elegidas al azar, sino las reconocidas como propias de un lenguaje no verbal universal (Matsumoto, Frank \& Hwang, 2016)

6 He de recalcar que las emociones están acompañadas de ideas y ciertos modos de pensamiento. Es decir, hay correlación entre las ideas y las emociones, pero no hay causalidad intrínseca, sino asociación mental contingente de ciertos pensamientos y emociones.

7 He decidido optar por afirmar que la representación mental crea la emoción y no que la realidad puede crear la emoción. La percepción supone una determinada carga semántica; que esta sea real o no, es irrelevante para considerar el surgimiento de la emoción. En tal sentido, opto por una descripción budista (Sanghakárshita, 2011) sobre el fenómeno de la percepción: la mente es la acción de producir una percepción, no una mímesis pasiva ni neutral de la realidad.

8 Incluso Mario Bunge, férreo enemigo del psicoanálisis, aceptó que el inconsciente es el máximo logro freudiano en Filosofía de la psicología (1988). No obstante, sobre lo que sí hay debate es si el inconsciente solamente concierne a procesos automáticos simples o a procesos dinámicos complejos (es distinto afirmar, por ejemplo, que cada vez que alguien ve a una persona atractiva se sonroja que esta misma tenga una fijación con personas muy parecidas 
físicamente a su madre que tienen habitualmente el carácter del padre). Este asunto será abordado a continuación.

9 No entraremos en el debate animalista en este punto, pero se puede deducir que la representación mental no es exclusiva del humano.

10 Nuestras representaciones mentales (usando este término à la Fodor) provienen tanto de nuestra capacidad de representar por imágenes, por sonidos, por tacto, por olfato, por sabor o por lenguaje.

11 Esto ya fue mencionado en los anteriores apartados, pero acá se explicará a partir de autores tradicionalmente asociados con la filosofía.

12 Respecto a este punto, nos puede ser de utilidad el debate que explica Gallagher (2018). En este, participaron H. L. Dreyfus y J. McDowell. La pregunta que se hacen es la siguiente: ¿el lenguaje lingüístico es el principal componente de nuestra mente? Dreyfus señala, por un lado, que la racionalidad está íntimamente relacionada con la comunicación entre humanos (dar y pedir razones), por lo que el componente lingüístico es necesario. Por otro lado, McDowell asegura que no hay necesidad de que ocurra ello, pese a que, en general, nuestras experiencias son susceptibles de ser conceptualizadas lingüísticamente. De hecho, esta última aseveración es la intención de Davidson, pero cree que el parecido que hay entre nuestra capacidad lingüística y la actitud proposicional lo autoriza a suponer que tenemos actitudes proposicionales por tener esta capacidad. No obstante, no se sigue uno de otro. El que la lingüística se parezca a la lógica no supone que sean lo mismo.

13 Esto ya fue afirmado en el segundo apartado, donde se establece que los bebés humanos y primates comprenden lógicamente la realidad sin poseer lenguaje.

14 Esta idea tiene una relación estrecha con la idea de "embodied rationality". Gallagher (2018) explica que los movimientos del cuerpo, sin una consciencia total de su realización, son parte del mismo hecho de que conozcamos el mundo. Es decir, conocemos la realidad mediante la acción. No obstante, parecería que se distancia de las ideas de Fodor: "On the enactivist view, the world is laid out in perception, not in terms of a conceptual, or proto-conceptual meaning, but first of all, in terms of differentiations that concern my action possibilities or affordances - the object is something I can reach, or not; something I can lift, or not; something I can move or not" ("Bajo el punto de vista enactivista, el mundo está dispuesto por la percepción, no en términos conceptuales ni proto-conceptuales, pero primero en términos de diferenciaciones que conciernen mis posibilidades de acción o asequibilidad -el objetivo es algo que puedo alcanzar o no, algo que puedo alzar o no, algo que pueda mover o no"; traducción nuestra) (Gallagher, 2018, p. 87). Parecería, en este caso, que se distancia de la actitud proposicional. No obstante, nos parece que la distancia no es tan amplia: la idea de "concepto" de Fodor (ver el siguiente pie de página) es bastante extensa y, en realidad, solo consiste en asumir que representamos el mundo proposicionalmente (la actitud proposicional es un vestigio de tener un "concepto"), pero esa representación no se restringe al acto de pensar lingüísticamente o "ver imágenes" en el cerebro, sino de conocer con todos los sentidos. Conocemos complejamente la realidad; luego actuamos utilizando ese conocimiento.

15 Este asunto es sumamente delicado, pues Fodor incluso explica que existen "conceptos" en la mente, pero no son lingüísticos y podrían ser representaciones mentales en un sentido sumamente laxo (ni siquiera son imágenes). En tal sentido, Fodor apuesta por suponer que el cerebro comprende la realidad en partes totalmente identificables (si uno puede entender qué es una vaca marrón se debe a que sabe qué es vaca y qué es marrón) (Fodor, 1998, p. 11). Dennett no estaría de acuerdo con esto, precisamente por otorgar una explicación totalmente lingüística a un fenómeno del cual no sabemos tanto. No obstante, de nuevo, 
la idea de "representación mental" de Fodor es tan laxa que se puede equiparar a decir "el cerebro representa la realidad", lo cual puede ser con olores, imágenes, gusto, tacto, sonidos u otra forma que no conocemos. La obstinación de este en utilizar la palabra "concepto" o descomponer las creencias como si estas fueran actitudes proposicionales en un sentido lingüístico es lo que, me parece, Dennett desafiaría.

16 Para una referencia neurocientífica, se puede revisar el trabajo de Anil Seth en su Ted Talk, Your brain hallucinates your conscious reality, o el libro 30-second brain.

\section{Referencias bibliográficas}

Bennett, M. R., \& Hacker, P. (2003). Philosophical foundations of neurosciences. Oxford: Blackwell.

Damasio, A. (1994). Descarte's error: emotion, reason and the human brain. Londres: Routledge.

Damasio, A. (2010). Self comes to mind: constructing the conscious brain. New York: Pantheon Books.

Davidson, D. (1982). Paradoxes of Irrationality. En D. Davidson, Problems of Rationality (pp. 169-187). Oxford: Oxford University Press (2004).

Davidson, D. (1990a). Epistemology externalized. En D. Davidson, Subjective, intersubjective, objective (pp. 193-205). Oxford: Clarendon Press (2001).

Davidson, D. (1990b). Representation and Interpretation. En D. Davidson, Problems of Rationality (pp. 87-99). Oxford: Oxford University Press (2004)

Davidson, D. (1998). The myth of the subjective. En D. Davidson, Subjective, intersubjective, objective (pp. 39-53). Oxford: Clarendon Press (2001).

Davidson, D. (2001). What Thought Requires. En D. Davidson, Problems of Rationality (pp. 135-149). Oxford: Oxford University Press (2004).

Dennett, D. (1969). Content and consciousness. Londres: Routledge

Dennett, D. (1991). Consciousness explained. New York: Back Bay Books

Eagle, M. (2018). Core Concepts in Classical Psychoanalysis. New York: Routledge

Elster, J. (1999) Alchemies of the mind: rationality and the emotions. Cambridge: Cambridge University Press

Fodor, J. (1992). A theory of content and other essays. Massachusetts: MIT Press.

Fodor, J. (1998). Concepts. Oxford: Clarendon Press Oxford.

Fodor, J. (2008). LOT 2 The Language of Thought Revised. Oxford: Clarendon Press Oxford.

Gabriel, M. (2017). I am not a brain. Massachusetts: Polity Press.

Gabriel, M. \& Searle, J. [ENCUENTROS DE FILOSOFÍA INTERCULTURA CANARIAS] (2018, diciembre 8) Debate posterior la conferencia magistral de MARKUS GABRIEL [Video]. Recuperado de: https://www.youtube. com/watch?v=sMYYXi4Qw9w 
Gabriel, M. (2018). Neo-existentialism: how to conceive of the human mind after Naturalism's failure. Massachusetts: Polity Press.

Gallagher, S. (2018). Embodied rationality. En G. Bronner, \& F. Di Loro, The mistery of rationality: mind, beliefs and the social sciences (pp. 83-94). Paris: Springer.

Goleman, D. \& Davidson, R. (2017). Altered traits. Londres: Routledge

Gomez, J. (2004). Apes, Monkeys, Children and the Growth of Mind. Massachusetts: Harvard University Press.

Gopnik, A., Meltzoff, A., \& Kuhl, P. (2001). The scientist in the crib. New York: Harper Collins.

Matsumoto, D., Frank, M. G., \& Hwang, H. S. (Eds.). (2013). Nonverbal communication: Science and applications. Thousand Oaks, CA, US: Sage Publications, Inc.

Mingyur Rinpoché. [Mindfulness 360-Center for Mindfulness] (2017, noviembre 21) "Meditation \& Monkey Mind" Yongey Mingyur Rinpoche [Video]. Recuperado de: https:/www.youtube.com/watch?v=ksp3iSUDqfo

Hernández, M. (1994). Sobre la irracionalidad en Donald Davidson. Contextos, 245263.

Livet, P. (2018). Dynamics of rationality and dynamics of emotions. En G. Bronner, \& F. Di Loro, The mystery of rationality: mind, beliefs and the social sciences (pp. 147-163). Paris: Springer.

Padmasiri, M. (1979). An Introduction to Buddhist Psychology. Londres: The MacMillan Press.

Quintanilla, P. (2014). Los límites de la irracionalidad. En B. Bettocci, \& R. Fatule, Una visión binocular. Psicoanálisis y Filosofía (pp. 123-137). Lima: Fondo Editorial de la Pontificia Universidad Católica del Perú.

Quintanilla, P. (2019) La comprensión del otro: explicación, interpretación y racionalidad. Lima: Fondo Editorial de la Pontificia Universidad Católica del Perú.

Rescorla, M. (21 de junio de 2019). The language of thought hypothesis. The Stanford Encyclopedia of Philosophy, Edward N. Zalta (ed.), Obtenido de https://plato. stanford.edu/entries/language-thought/.

Rochat, P. (2001). The infant's world. Massachusetts: Harvard University Press.

Sangharákshita, U. (2011 [1998]). La mente en la psicologia budista: explorando la dimensión psicológica en la ética budista a través de los textos canónicos del Abhidharma. Mexico DF: Ediciones CBCM.

Sapolsky, R. (2011, 1 de febrero). 23. Language. Stanford, California, Estados Unidos [Video]. Recuperado de https://www.youtube.com/watch?v=SIOQgY1tqrU\&t=

Sapolsky, R. (2017) Behave:the biology of humans at our best and worst. New York: Penguin Press. 
Searle, J. (2007). Freedom and Neurobiology: Reflections on Free Will, Language, and Political Power. New York: Columbia University Press

Schwitzgebel, E. (21 de marzo de 2015). Stanford Encyclopedia of Philosophy. Obtenido de https://plato.stanford.edu/archives/sum2015/entries/belief/

Solms, M. [MacLean Center] (2015, octubre 20) Mark Solms, PhD - Psychoanalysis \& Neuroscience. [Video]. Recuperado de: https://www.youtube.com/watch?$\mathrm{v}=\mathrm{Rv} 9 \mathrm{jw} \mathrm{CDNO} \mathrm{M}$

Solms, M. (2017). What is 'the unconscious,' and where is it located in the brain? A neuropsychoanalytic perspective. Ann. NY. Acad. Sci. 1406, 90-97.

Solms, M. (2018). The scientific standing of psychoanalysis. Br. J. Psychiatry Int. 15, 5-8. doi: 10.1192/bji.2017.4

Solms, M. \& Pankseppl, J. (2015). The "id" knows more than the "ego" admits. En Solms, M., The feeling brain (pp. 143-181). London: Karnac.

Solms, M. \& Turnbull, O. (2015). What is neuropsychoanalysis?. En Solms, M., The feeling brain (pp. 13-34). London: Karnac.

Yalowitz, S. (12 de diciembre de 2014). Anomalous monism. Stanford Encyclopedia of Philosophy. Obtenido de https://plato.stanford.edu/entries/anomalous-monism/ 